\title{
Hierarchical GPC-Based PID Control Strategy for SST of USC under Variable Loads
}

\author{
Xin Li, Yanjun Fang and Rong Zhang \\ Department of Automation, Wuhan University, China \\ xinli1005@whu.edu.cn
}

\begin{abstract}
A hierarchical PID control strategy based on generalized predictive control (GPC) algorithm is presented to solve the model uncertainty of the superheated steam temperature (SST) and the application problem of advanced control algorithms in distributed control system (DCS) of the ultra-supercritical (USC) units. The upper level is based on generalized predictive control algorithm, which also has the functions of model identification and PID parameters tuning. The conventional cascade PID control strategy is applied in the bottom level. Simulations carried out with the field operation data from Chaozhou USC units. The simulation results show that the hierarchical predictive PID control system is qualified with strong stability and robustness, which can adapt to the model change process of the SST control.
\end{abstract}

Keywords: USC, SST, Hierarchical Structure, GPC, Model Identification

\section{Introduction}

With the development of power industry and the power grid capacity, the construction of supercritical and ultra-supercritical (USC) units is gradually accelerating [1]. The superheated steam temperature(SST) of the once-through boiler in a USC unit has complex features of great inertia, large delay, nonlinearity, time-varying and model uncertainty, which is affected by the ratio of fuel to water, intermediate point temperature, flow of the desuperheater spray water [2]. Usually, the ratio of fuel to water and the flow of the desuperheater spray water are controlled together in the SST control system, which means that the process of the desuperheater spray water adjustment is more complex since it needs to be faster and more precise [3]. Conventional SST control is combined with feedforward compensation, cascade system and some other strategies, applying fixed or piecewise PID parameters, but the influence of model change on the SST under variable loads has been ignored [4,5]. Manual operation and supervisory control are needed under complex loads conditions, which seriously affects the economy and safety of the units [6].

The existing improved PID algorithm has been successfully applied in some areas [7-8]. However, there are still some problems in thermal process control of power plants, such as complex structures, various parameter tuning rules [9]. As there is no effective support in the hardware and software for the process control design in distributed control system (DCS), the introduction of advanced control algorithms requires large-scale transformation of hardware structure [10]. Moreover, it is hard for the operators who are only familiar with PID parameters adjustment to understand the meaning of advanced control algorithms [11].

Aiming to solve the SST control problem of USC units under large-scale load changing, a hierarchical GPC-based PID control strategy is presented in this paper, which can realize the application of advanced control and intelligent optimization technique in DCS. The simulation results show that the proposed control algorithm is qualified with strong stability and robustness. 


\section{Hierachical Structure of SST Control System}

\subsection{Design of Hierarchical Structure}

The cascade PID strategy is the most widely used in the SST control system, in which the spray desuperheating system is usually used as minor loop to overcome the disturbance in time and improve the control effect[12]. But the cascade control doesn't has optimization strategy to adjust the PID parameters, which means the engineers have to design the PID parameters in advance to meet the needs of SST control when the load changes[13].

The SST control system proposed in this paper shown in Figure 1 has a hierarchical structure, in which $r(t)$ and $y(t)$ are the input and output respectively. The cascade PID control strategy, which is common used in DCS of the thermal power units, is applied in the bottom level to adapt to the characteristics of the control system effectively. There are only three control parameters, $\mathrm{K}_{\mathrm{p}}, \mathrm{K}_{\mathrm{i}}, \mathrm{K}_{\mathrm{d}}$, for engineers, which are easy for them to understand. The upper level consists of three modules - model parameters identification module, on-line optimization module and PID parameters tuning module. The performance index of generalized predictive control(GPC) is introduced, and the SST controlled model is identified online, and the parameters of the controller are rolling optimized. A data communication interface is needed to transfer the control variables and output data of the bottom level to the upper level, in which the three modules will operate to gain the updated PID parameters and control variables and then transfer them back to the bottom level to adjust the parameters of the controller. In this way, the field engineers do not need to understand the advanced algorithm in the upper level, and large-scale transformation of hardware structure is not necessary in the DCS.

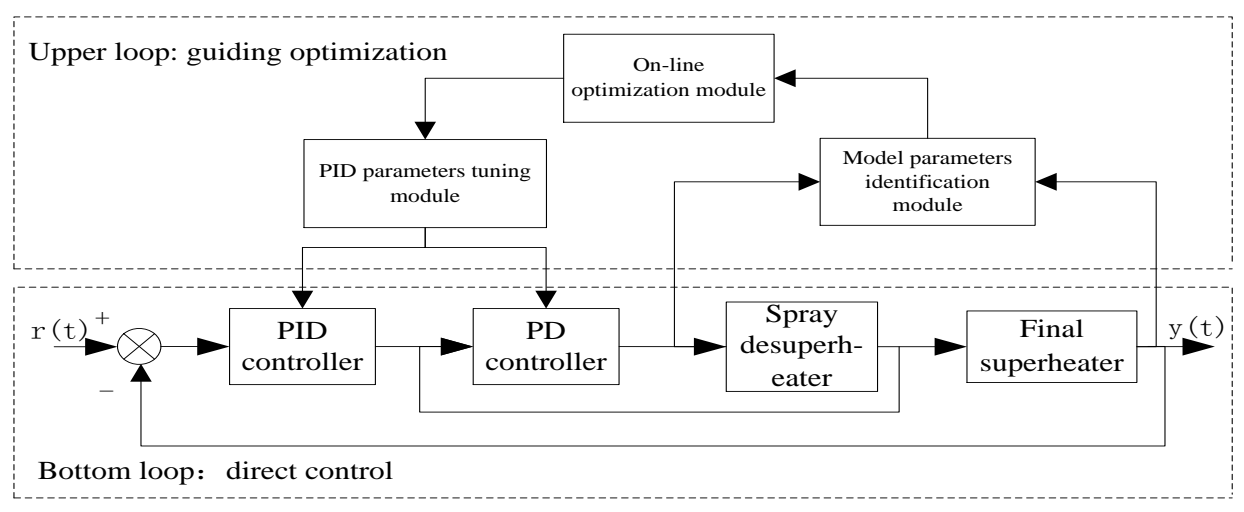

Figure 1. Hierarchical Structure of SST Control System

\subsection{Soft Switching of the Upper level}

In order to adapt to the model change process of the SST control as well as decrease the operation cost, a sort of soft switching strategy is employed to control the operation of the upper level. There are three operation states based on the load and control quality changes, which can be expressed as

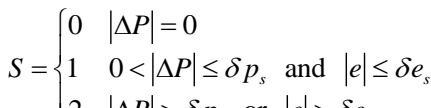

$$
\begin{aligned}
& 2|\Delta P|>\delta p_{s} \text { or }|e|>\delta e_{s}
\end{aligned}
$$

where $\mathrm{S}$ is state of the soft switching, $\Delta P$ is the load variation, $|e|$ is the error of the main loop of cascade PID control system, $\delta p_{s}$ is the given critical value of working condition, and $\delta e_{s}$ is the value of error. 
1) When the load is steady $(\Delta P=0)$, the SST controlled model can be seen as certain, which means the PID parameters adjusted before can guarantee the stability of SST control system. Therefore, the upper level will not work $(S=0)$, and the SST control system equals to cascade PID control system.

2) When the load changes a little $\left(0<|\Delta P| \leq \delta p_{s}\right)$, and the control quality doesn't change dramatically $\left(|e| \leq \delta e_{s}\right)$, the change of the SST controlled model won't make great impact on control effect, so that the PID parameters adjusted before are still available. But in order to prevent that the PID parameters won't adapt to the change in the future, the model parameters identification module of the upper level should start to work $(S=1)$.

3) When the load changes greatly $\left(|\Delta P|>\delta p_{s}\right)$, or the control quality changes dramatically $\left(|e|>\delta e_{s}\right)$, the SST controlled model will change obviously, and the PID parameters adjusted before is unavailable. So it's necessary to make the upper level work $(S=2)$, including all of the three modules.

\section{Control Strategy of Upper Level}

The operation of the upper level includes three stages. Stage One, initialization, including setting parameters and calculating the initial values. Stage Two, identify the mathematical model of the controlled object. Stage Three, figure out the new PID parameters for the bottom level. The details are as follows.

\subsection{GPC Algorithm}

The conventional cascade PID controller for SST of USC units uses incremental controller [14], which can be described as

$$
\Delta u(k)=w_{0} e(k)+w_{1} e(k-1)+w_{2} e(k-2)
$$

where $\omega_{0}=K_{p}+K_{i}+K_{d}, \omega_{1}=-K_{p}-2 K_{d}, \omega_{2}=K_{d}$, and $\mathrm{K}_{\mathrm{p}}, \mathrm{K}_{\mathrm{i}}, \mathrm{K}_{\mathrm{d}}$ are the proportion coefficient, integral coefficient, and differential coefficient respectively. $e(k)$ is the error between system output and the set point at $k$ moment. Equation (2) can also be expressed as

$$
\Delta u(k)=\sum_{i=0}^{2} w_{i}(k) e(k-i)=\boldsymbol{W}^{T}(k) \boldsymbol{e}(k)
$$

where $\boldsymbol{W}^{T}(k)=\left[\omega_{0}(k) \omega_{1}(k) \omega_{2}(k)\right], \boldsymbol{e}(k)=\left[e_{0}(k) e_{1}(k) e_{2}(k)\right]$.

The simplified controlled auto-regressive integrated moving average(CARIMA) model in GPC theory is introduced to describe the object with random disturbance as follows[15].

$$
A\left(z^{-1}\right) y(k)=B\left(z^{-1}\right) u(k-1)+\xi(k) / \Delta
$$

where $u(k)$ is the control variable, $\xi(k)$ is the white noise, $\Delta=1-\mathbf{z}^{-1} . A\left(z^{-1}\right), B\left(z^{-1}\right)$ are the weighted polynomial of controlled object and the disturbance object, which can be described as

$$
\left\{\begin{array}{l}
A\left(z^{-1}\right)=1+a_{1} z^{-1}+a_{2} z^{-2}+\ldots+a_{n_{a}} z^{-n_{a}} \\
B\left(z^{-1}\right)=b_{0}+b_{1} z^{-1}+b_{2} z^{-2}+\ldots+b_{n_{b}} z^{-n_{b}}, b \neq 0
\end{array}\right.
$$

From (3) and (4), the CARIMA model can be presented as

$$
\left(1-z^{-1}\right) A\left(z^{-1}\right) y(k)=B\left(z^{-1}\right) \boldsymbol{W}^{T}(k) \boldsymbol{e}(k)+\xi(k)
$$

Diophantine Equation is introduced to predict the output $j$ steps early as follows.

$$
1=E_{j}\left(z^{-1}\right) A\left(z^{-1}\right) \Delta+z^{-j} F_{j}\left(z^{-1}\right)
$$


where $E_{j}\left(z^{-1}\right)=e_{j 0}+e_{j 1} z^{-1}+\ldots+e_{j, j-1} z^{-j+1}, e_{j 0}=1$, and $F_{j}\left(z^{-1}\right)=f_{j 0}+f_{j 1} z^{-1}+\ldots+f_{j n} z^{-n}$

Through solving Diophantine Equation by using GPC algorithm, the optimal output predicted $j$ steps early is calculated as

$$
\begin{aligned}
& \hat{y}(k+j)=G_{j}\left(z^{-1}\right) \Delta u(k+j-1)+F_{j}\left(z^{-1}\right) y(k)+H_{j}\left(z^{-1}\right) \Delta u(k-1) \\
& =G_{j}\left(z^{-1}\right)\left(\sum_{i=0}^{2} w_{i}(k+j-1) e(k+j-i-1)\right)+F_{j}\left(z^{-1}\right) y(k)+H_{j}\left(z^{-1}\right) \Delta u(k-1)
\end{aligned}
$$

The vector format of (8) can be described as

$$
\hat{\boldsymbol{Y}}=\boldsymbol{G} \Delta \boldsymbol{U}+\boldsymbol{f}
$$

where

$$
\hat{\boldsymbol{Y}}=[y(k+1), y(k+2), \ldots \ldots, y(k+n)]^{T},
$$

$$
\Delta \boldsymbol{U}=[\Delta u(k), \Delta u(k+1), \ldots \ldots, \Delta u(k+n-1)]^{T},
$$

$$
f=[f(k+1), f(k+2), \ldots \ldots, f(k+n)]^{T}=H \Delta u(k-1)+F y(k),
$$

$$
\begin{gathered}
G=\left[\begin{array}{cccc}
g_{0} & 0 & \cdots & 0 \\
g_{1} & g_{0} & \cdots & 0 \\
\vdots & \vdots & \ddots & \vdots \\
\vdots & \vdots & \cdots & g_{0} \\
g_{N-1} & g_{N-2} & \cdots & g_{N-N_{u}}
\end{array}\right]_{N \times N_{u}} \\
H^{T}=\left[H_{1}, \ldots \ldots, H_{N}\right], \quad F^{T}=\left[\begin{array}{ll}
F_{1} & \ldots . ., F_{N}
\end{array}\right] .
\end{gathered}
$$

From (8), the optimized performance index of GPC can be described as

$$
\min J(t)=\sum_{J=1}^{N}\left[L_{j}-G_{j} \boldsymbol{W}^{T}\left(k_{m}\right) \hat{\boldsymbol{e}}\left(k_{m}\right)\right]^{2}+\sum_{j=1}^{N_{u}} \lambda_{j}\left[\boldsymbol{W}^{T}\left(k_{m}\right) \hat{\boldsymbol{e}}\left(k_{m}\right)\right]^{2}
$$

where $N$ is the optimization time domain, $N_{u}$ is the control time domain which means the control variables won't change after $N_{u}$ steps. $L_{j}=\omega(k+j)-H_{j} \Delta u(k-1)-F_{j} y(k), \lambda_{j}$ is a positive constant, $G_{j}\left(z^{-1}\right)=E_{j}\left(z^{-1}\right) B\left(z^{-1}\right)$.

Based on the performance index function, the optimal control law can be calculated as

$$
\boldsymbol{u}=\left(\lambda \boldsymbol{I}+\boldsymbol{G}^{T} \boldsymbol{G}\right)^{-1} \boldsymbol{G}^{T}[\boldsymbol{\omega}-\boldsymbol{F} y(k)-\boldsymbol{H} \Delta u(k-1)]
$$

From (10), $\Delta k$ can be described as

$$
\hat{\boldsymbol{W}}=\left[\hat{\boldsymbol{e}}^{T}\left(\boldsymbol{G}^{T} \boldsymbol{G}+\lambda \boldsymbol{I}\right) \hat{\boldsymbol{e}}\right]^{-1}\left(\hat{\boldsymbol{e}} \boldsymbol{G}^{T} \boldsymbol{L}\right)
$$

Therefore, the optimal control sequence is calculated as

$$
\Delta \boldsymbol{u}(k)=\left[\begin{array}{c}
\Delta u(k) \\
\Delta u(k+1) \\
\vdots \\
\Delta u\left(k+N_{u}-1\right)
\end{array}\right]=\left[\begin{array}{c}
\boldsymbol{W}^{T}(k) \boldsymbol{e}_{1}(k) \\
\boldsymbol{W}^{T}(k+1) \boldsymbol{e}_{2}(k+1) \\
\vdots \\
\boldsymbol{W}^{T}\left(k+N_{u}-1\right) \boldsymbol{e}_{N_{u}}\left(k+N_{u}-1\right)
\end{array}\right]=\hat{\boldsymbol{e}} \hat{\boldsymbol{W}}
$$

where 


$$
\begin{aligned}
& \hat{\boldsymbol{e}}=\left[\begin{array}{cccccccccc}
e_{1}(k) & e_{1}(k-1) & e_{1}(k-2) & 0 & 0 & 0 & \ldots & 0 & 0 & 0 \\
0 & 0 & 0 & e_{2}(k) & e_{2}(k-1) e_{2}(k-2) & \vdots & 0 & 0 & 0 \\
\vdots & \vdots & \vdots & \vdots & \vdots & \vdots & \vdots & \vdots & \vdots & \vdots \\
0 & 0 & 0 & 0 & 0 & 0 & \ldots & e_{N_{u}}(k) & e_{N_{u}}(k-1) & e_{N_{u}}(k-2)
\end{array}\right]_{N_{u} \times 3 N_{u}}, \\
& \hat{\boldsymbol{W}}=\left[\begin{array}{c}
\boldsymbol{W}(k) \\
\boldsymbol{W}(k+1) \\
\vdots \\
\boldsymbol{W}\left(k+N_{u}-1\right)
\end{array}\right]=\left[\begin{array}{ccc}
{\left[w_{0}(k)\right.} & w_{1}(k) & \left.w_{2}(k)\right]^{T} \\
{\left[w_{0}(k+1)\right.} & w_{1}(k+1) & \left.w_{2}(k+1)\right]^{T} \\
\vdots & \\
{\left[w_{0}\left(k+N_{u}-1\right)\right.} & w_{1}\left(k+N_{u}-1\right) & \left.w_{2}\left(k+N_{u}-1\right)\right]^{T}
\end{array}\right]_{3 N_{u} \times 1}
\end{aligned}
$$

From (12), the optimal PID parameters are obtained, as shown in (14)

$$
\left\{\begin{array}{l}
k_{p}(k)=-w_{1}(k)-2 w_{2}(k) \\
k_{i}(k)=w_{0}(k)+w_{1}(k)+w_{2}(k) \\
k_{d}(k)=w_{2}(k)
\end{array}\right.
$$

\subsection{Model Parameters Identification}

The method based on forgetting factor recursive least square(FFRLS) is introduced in this part to identify the controlled object model of SST. In the control process, the parameters need to be figured out using CARIMA model instead of directly identifying the time constant, proportion coefficient and delay time [16]. The controlled object model of minor loop and main loop in least square format based on CARIMA can be described as

$$
\begin{gathered}
\left\{\begin{array}{l}
y_{2}(k)=\boldsymbol{\varphi}_{2}^{T}(k) \boldsymbol{\theta}_{2}+\xi_{2}(k) / \Delta \\
y_{1}(k)=\boldsymbol{\varphi}_{1}^{T}(k) \boldsymbol{\theta}_{1}+\xi_{1}(k) / \Delta
\end{array}\right. \\
\text { where } \\
\left\{\begin{array}{l}
\boldsymbol{\varphi}_{2}(k)=\left[-y_{2}(k-1), \ldots,-y_{2}\left(k-n_{a}^{2}\right), u_{2}(k-1), \ldots, u_{2}\left(k-n_{b}^{2}-1\right)\right] \\
\boldsymbol{\theta}_{2}=\left[a_{1}^{2}, \ldots, a_{n_{a}^{2}}^{2}, b_{0}^{2}, \ldots, b_{n_{b}^{2}}^{2}\right]
\end{array}\right. \\
\left\{\begin{array}{l}
\boldsymbol{\varphi}_{1}(k)=\left[-y_{1}(k-1), \ldots,-y_{1}\left(k-n_{a}^{1}\right), u_{1}(k-d), \ldots, u_{1}\left(k-n_{b}^{1}-d\right)\right] \\
\boldsymbol{\theta}_{1}=\left[a_{1}^{1}, \ldots, a_{n_{a}^{1}}^{1}, b_{0}^{1}, \ldots, b_{n_{b}^{1}}^{1}\right]
\end{array}\right.
\end{gathered}
$$

The parameter matrix updates automatically every once in $N_{p}$ sampling time, and the identification proceeds over time, by which the on-line data sampled forms a rolling window. Let $N_{d}$ be sample space, $p=N_{d} / N_{p}$, and $N_{d}>N_{p}$.

The process of FFRLS model identification of SST in rolling window can be described as follows.

Step1: Set sampling space $N_{d}$ and sampling time $N_{p}$, and ensure $N_{d} / N_{p}$ is an integer, $N_{d}>N_{p}$.

Step2: Determine the initial value $\theta(0)$ and $\boldsymbol{P}(0)$, and set forgetting factor $\rho$, and obtain the initial data.

Step3: Obtain current output $y(k)$ and input $u(k)$ through sampling, and save it to the data vector of rolling window.

Step4: Obtain $\boldsymbol{K}(k), \theta(k)$ and $\boldsymbol{P}(k)$ by using FFRLS. The formulas for estimating parameters of FFRLS is

$$
\left\{\begin{array}{l}
\boldsymbol{\theta}(\boldsymbol{k})=\boldsymbol{\theta}(\boldsymbol{k}-1)+\boldsymbol{K}(\boldsymbol{k})\left[\boldsymbol{y}(\boldsymbol{k})-\boldsymbol{\varphi}^{\mathrm{T}}(\boldsymbol{k}) \boldsymbol{\theta}(\boldsymbol{k}-1)\right] \\
\boldsymbol{K}(\boldsymbol{k})=\boldsymbol{P}(\boldsymbol{k}-1) \boldsymbol{\varphi}(\boldsymbol{k})\left[\rho+\boldsymbol{\varphi}^{\mathrm{T}}(\boldsymbol{k}) \boldsymbol{P}(\boldsymbol{k}-1) \boldsymbol{\varphi}(\boldsymbol{k})\right]^{-1} \\
\boldsymbol{P}(\boldsymbol{k})=\frac{1}{\rho}\left[\boldsymbol{I}-\boldsymbol{K}(\boldsymbol{k}) \boldsymbol{\varphi}^{\mathrm{T}}(\boldsymbol{k})\right] \boldsymbol{P}(\boldsymbol{k}-1)
\end{array}\right.
$$


where $\varphi$ is the observation data.

Step5 : $k \rightarrow k+1$, return to Step2, and conduct circular computations in rolling window.

After the model parameters of SST is identified on-line in rolling window using FFRLS, the results can be transferred to the on-line optimization module for the next step of computation.

\subsection{Model of PID Parameters Tuning}

The hierarchical predictive PID control strategy adopts optimal control law, and the optimal control sequence is obtained by calculating minimized GPC performance index, which can be used to figure out the PID parameters. To simplify the process, the problem of obtaining the constraint control variables is transformed into a problem of tuning PID parameters. The mathematical model of the main loop parameters tuning is as follows.

$$
\begin{gathered}
\min J_{1}(t)=E\left\{\sum_{J=N_{11}}^{N_{12}}\left[y_{1}(t+j)-\omega_{1}(t+j)\right]^{2}+\sum_{j=1}^{N_{11}} \lambda_{1}(j)\left[\Delta u_{1}(t+j-1)\right]^{2}\right\} \\
\text { s.t. } \quad \hat{\boldsymbol{\kappa}}_{1} \in S_{1} \\
u_{1 \min } \leq u_{1}(t+j) \leq u_{1 \max } \\
\Delta u_{1 \min } \leq \Delta u_{1}(t+j) \leq \Delta u_{1 \max }
\end{gathered}
$$

where Subscript 1 represents the parameters of main loop, $J_{1}$ is the GPC performance index of main loop, $\hat{\kappa}_{1}=\left[K_{p 1}, K_{i l}, K_{d 1}\right]^{T}$ is PID parameter vectors of main loop, and $s_{1}$ is the custom zone of main loop PID parameters.

The mathematical model of minor loop parameters tuning is as follows.

$$
\begin{gathered}
\min J_{2}(t)=E\left\{\sum_{J=N_{21}}^{N_{22}}\left[y_{2}(t+j)-\omega_{2}(t+j)\right]^{2}+\sum_{j=1}^{N_{24}} \lambda_{2}(j)\left[\Delta u_{2}(t+j-1)\right]^{2}\right\} \\
\text { s.t. } \quad \hat{\boldsymbol{\kappa}}_{2} \in S_{2} \\
u_{2 \min } \leq u_{2}(t+j) \leq u_{2 \max } \\
\Delta u_{2 \min } \leq \Delta u_{2}(t+j) \leq \Delta u_{2 \max }
\end{gathered}
$$

where Subscript 2 represents the parameters of minor loop, $J_{2}$ is the GPC performance index of minor loop, $\hat{\boldsymbol{\kappa}}_{2}=\left[K_{p 2}, K_{d 2}\right]^{T}$ is PD parameter vectors of minor loop, and $s_{2}$ is the custom zone of minor loop PD parameters.

The conventional methods of PID parameters tuning aren't easy to realize in computers and the robustness of the system isn't strong enough. The fixed parameters don't adapt to the model change process of the SST control. To meet the needs of hierarchical predictive PID control and solve the constraint control variables, intelligence algorithms, which have high precision, high speed, and strong robustness, can be introduced, such as genetic algorithm, particle swarm optimization, immune algorithm, etc.[17,18]. But this is not the topic of this paper and can be further studied.

\section{Simulation and Analysis}

\subsection{Establishment of the Multi-model Set of SST}

In order to obtain the linear model set of SST control object, which is required in simulation experiments, a dynamic model identification method of superheater in USC is used based on multi-model modeling theory under variable loads. The field operation data of 3\# Unit of Chaozhou USC, China, is used in the identification process of model parameters. Four typical loads, 1000MW, $850 \mathrm{MW}, 700 \mathrm{MW}$ and 550MW, are selected, and the models of spray desuperheater(leading segment) and final superheater(inert segment) under variable loads conditions are established. The SST multi-model set of of Chaozhou USC is obtained after repeated identification tests, and is shown in Table 1. The multi-model set is used to produce data during the process of model identification in the simulation. 
Table 1. SST Muti-Model Set of Chaozhou USC

\begin{tabular}{ccc}
\hline Load & $\begin{array}{c}\text { Leading } \\
\text { Segment } W W_{2}(s)\end{array}$ & Inert Segment $W_{1}(s)$ \\
\hline $\begin{array}{c}100 \%- \\
1000 \mathrm{MW}\end{array}$ & $-0.332 /(7.99 s+1)^{2}$ & $1.119 e^{-48 s} /(99.84 s+1)$ \\
$90 \%-900 \mathrm{MW}$ & $-0.512 /(15.51 s+1)^{2}$ & $1.116 e^{-70 s} /(105.3 s+1)$ \\
$70 \%-700 \mathrm{MW}$ & $-0.579 /(21.86 s+1)^{2}$ & $1.111 e^{-90 s} /(120.5 s+1)$ \\
$50 \%-500 \mathrm{MW}$ & $-1.055 /(29.89 s+1)^{2}$ & $1.107 e^{-122 s} /(163.9 s+1)$ \\
\hline
\end{tabular}

\subsection{Parameters Setting}

Simulink is used to construct the simulation platform of SST control system, and the system model is divided into a two-level structure in accordance with the design of SST control system. The bottom level contains the SST cascade PID control, the multi-model set, white noise, etc. The upper level contains FFRLS algorithm, Ziegler-Nichols (Z-N) algorithm, etc. The dynamic process of SST control system is affected by the model precision and control parameters. Therefore, the parameters of control system need to be set before the simulation test. The parameters are shown in Table 2.

Table 2. Parameters Setting of Hierachical Predictive PID

\begin{tabular}{|c|c|c|c|c|c|c|c|c|c|c|}
\hline \multirow{2}{*}{ Loop } & \multicolumn{5}{|c|}{ GPC Parameters } & \multicolumn{5}{|c|}{$\begin{array}{c}\text { FFRLS Rolling Identification } \\
\text { Parameters }\end{array}$} \\
\hline & $N_{1}$ & $N_{2}$ & $N_{u}$ & & $\alpha$ & & $\lambda$ & $N_{d}$ & 1 & $\rho$ \\
\hline Main & 1 & 10 & 1 & 6 & 0. & 5 & 0. & $0^{20}$ & 1 & 0.95 \\
\hline Minor & $\begin{array}{l}\mathrm{d}+ \\
10\end{array}$ & $2 d+10$ & 1 & 7 & 0. & 7 & 0. & $0^{60}$ & $\leq$ & 0.98 \\
\hline
\end{tabular}

Note: T is sampling period, $N_{l}, N_{2}$ are the starting and ending time of prediction, $\alpha$ is diffusion factor, $\mathrm{d}$ is inert segment delay which updates according to identification results.

In the simulation process, the proposed control strategy is compared with piecewise cascade PID control strategy. Both of them use the conventional Z-N algorithm to adjust PID parameters. Since piecewise PID control strategy has fixed parameters in each of the load segment, MATLAB pidtool is used for PID parameters tuning. The results are as Table 3.

Table 3. Parameters Setting of Piecewise PID

\begin{tabular}{cccccc}
\hline \multirow{2}{*}{ load segment } & Kp1 & Main Loop & \multicolumn{2}{c}{ Minor Loop } \\
& 0.7023 & 0.0152 & 3.2344 & 12.1393 & 17.1003 \\
\hline $95 \%-100 \%$ & 1.1032 & 0.0189 & 21.4209 & 17.0014 & 15.4571 \\
$90 \%-95 \%$ & 1.0027 & 0.0094 & 1.2451 & 22.1893 & 24.1025 \\
$80 \%-90 \%$ & 1.5038 & 0.0103 & 27.511 & 14.0612 & 10.1447 \\
$60 \%-80 \%$ & 0.6706 & 0.0052 & 14.879 & 7.3367 & 27.2208 \\
$40 \%-60 \%$ & & & & & \\
\hline
\end{tabular}




\subsection{Simulation Results}

1) Simulation under monotone load changes

In this part, the SST control simulation starts under 50\% load (or 90\% load), and runs for 20 minutes after the control system reaches a steady state. Then the load increases (or decreases) $1 \%$ per minute to $70 \%$ and also stays for 20 minutes. Finally the load increases (or decreases) $1 \%$ per minute to $90 \%$ (or $50 \%$ ) and lasts for 20 minutes. The variation trends of SST control under monotone increasing and decreasing load are shown in Figure 2 and Figure 5 respectively. Mid-point (60\% and 80\%) switch mode of transition load is applied to transform the model. The control simulation results of the two control strategies without disturbance and with internal disturbance are obtained, and the system response curves are shown in Figure 3, Figure 6 and Figure 4, Figure 7 respectively.

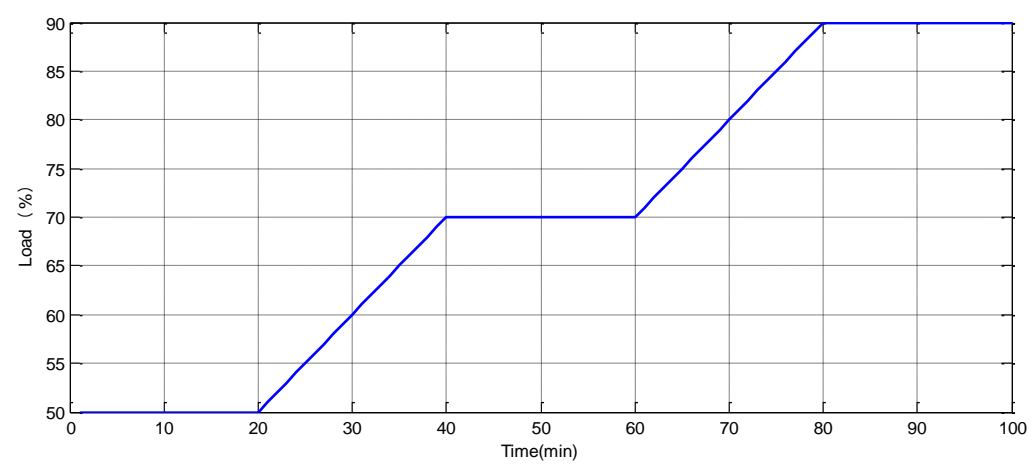

Figure 2. Variation Trend of Monotone Increasing Load

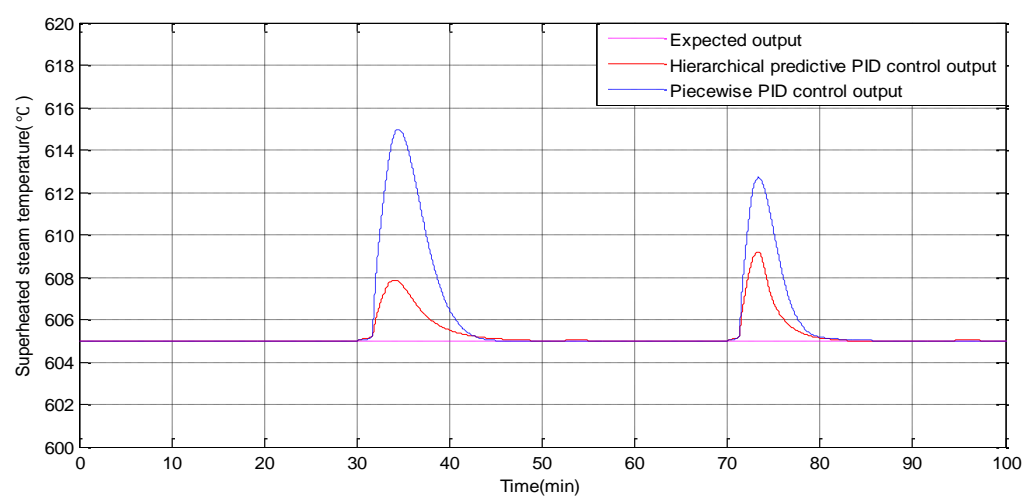

Figure 3. Output Curves of System Step Response without Disturbance under Monotone Increasing Load 


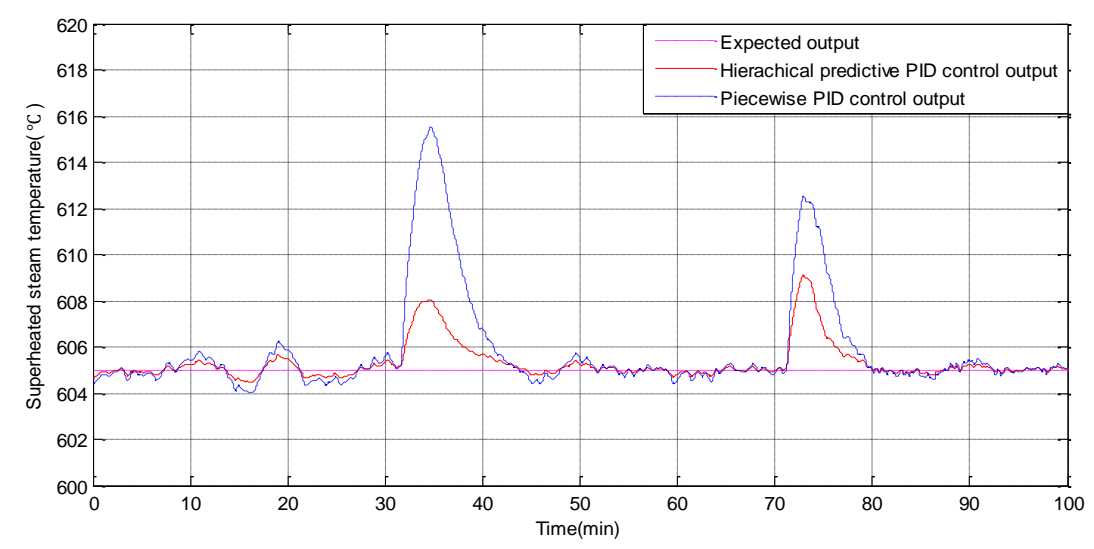

Figure 4. Output Curves of System Step Response with Internal Disturbance under Monotone Increasing Load

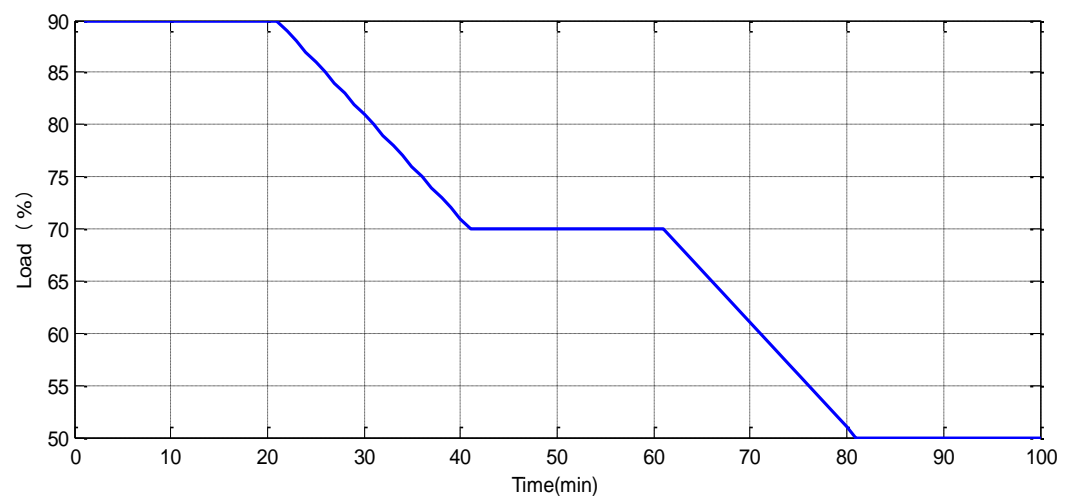

Figure 5. Variation Trend of Monotone Decreasing Load

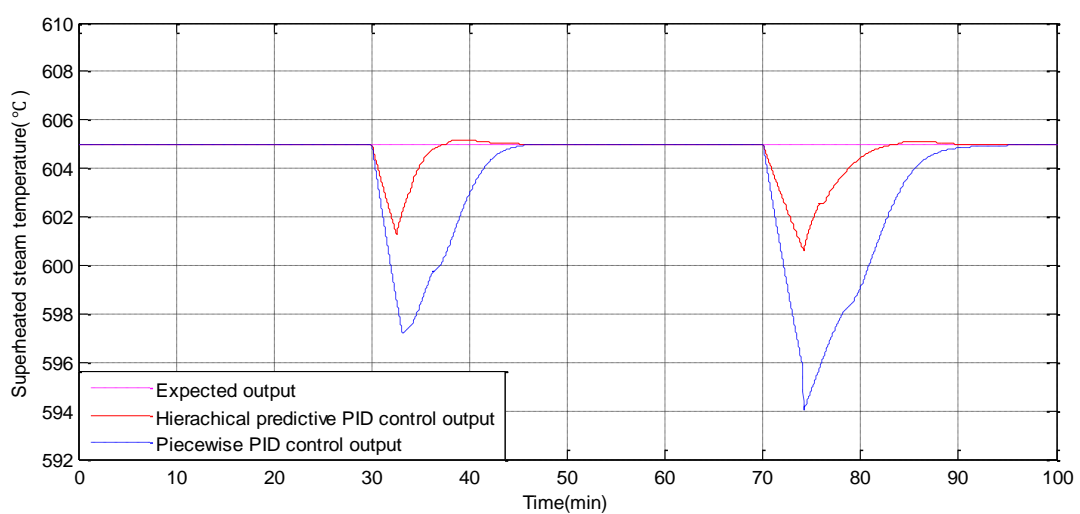

Figure 6. Output Curves of System Step Response without Disturbance under Monotone Decreasing Load 


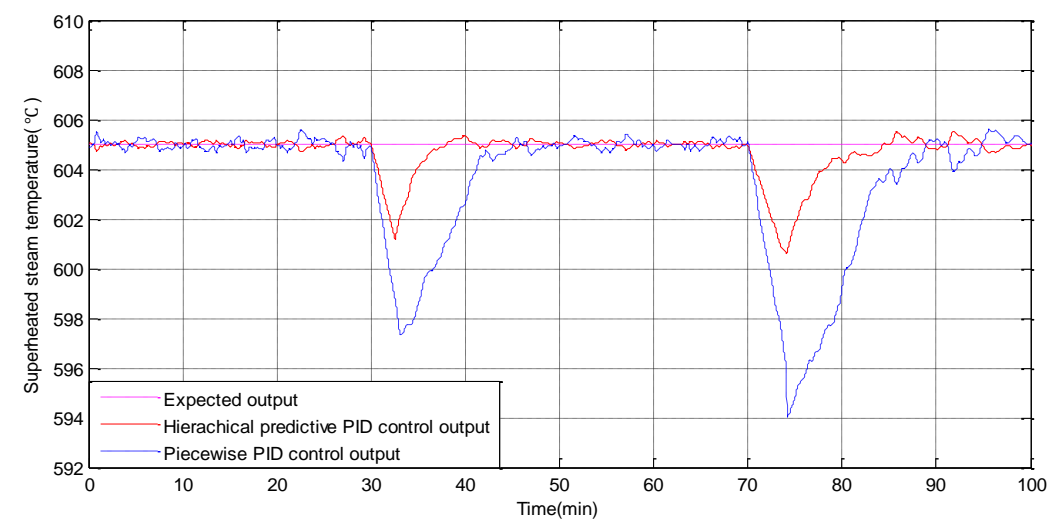

\section{Figure 7. Output Curves of System Step Response with Internal Disturbance under Monotone Decreasing Load}

It can be seen from Figure 3 and Figure 6 that the hierarchical predictive PID control strategy makes smaller overshoot in the load transition process, which means the proposed method enables SST control system to have a better control effect when the load changes monotonically. Then the white noise is introduced in the simulation whose expectation is 0 and variance is 400 . It can be seen from Figure 4 and Figure 7 that as the minor loop of the proposed structure is qualified with a better anti-disturbance ability, the introduction of internal disturbance doesn't cause a large fluctuation of SST. From Figure 6 and Figure 7, it can be seen that the proposed method makes the shorter in the load decreasing process. In conclusion, the hierarchical predictive PID control system has a better control performance and stronger anti-disturbance ability under monotone load changes.

\section{2) Simulation under non-monotone load changes}

In this part, the SST control simulation starts under $90 \%$ load (or 100\% load), and runs for 20 minutes after the control system reaches a steady state. Then the load increases (or decreases) $0.5 \%$ per minute to $100 \%$ (or $90 \%$ ) and also stays for 20 minutes. Finally the load decreases (or increases) $0.5 \%$ per minute to $90 \%$ (or 100\%) and lasts for 20 minutes. The variation trends of SST control under non-monotone load changes are shown in Figure 8 and Figure 11. Mid-point (95\%) switch mode of transition load is applied to transform the model. The control simulation results of the two control strategies without disturbance and with internal disturbance are obtained, and the system response curves are shown in Figure 9, Figure 12 and Figure 10, Figure 13 respectively.

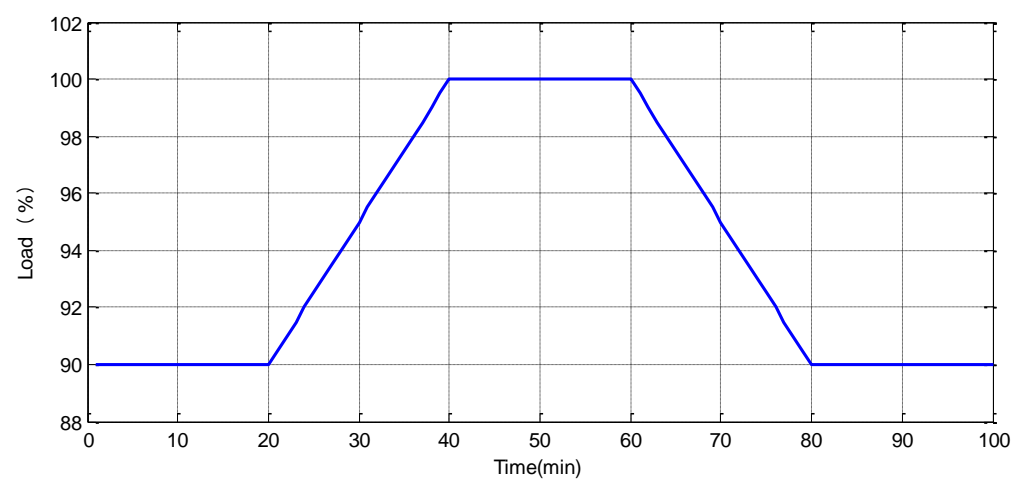

Figure 8. Variation Trend of Load that Increases First and Decreases Then 


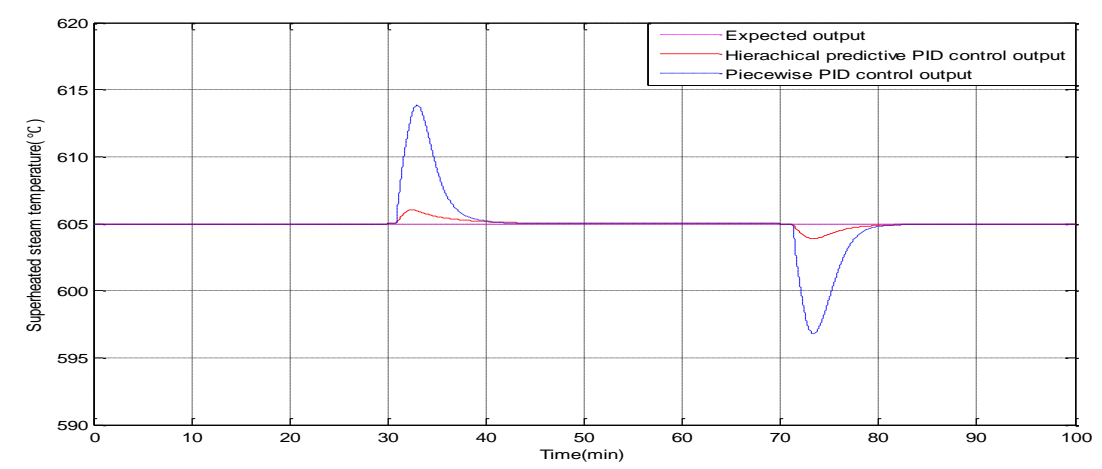

Figure 9. Output Curves of System Step Response without Disturbance under Load that Decreases First and Increases Then

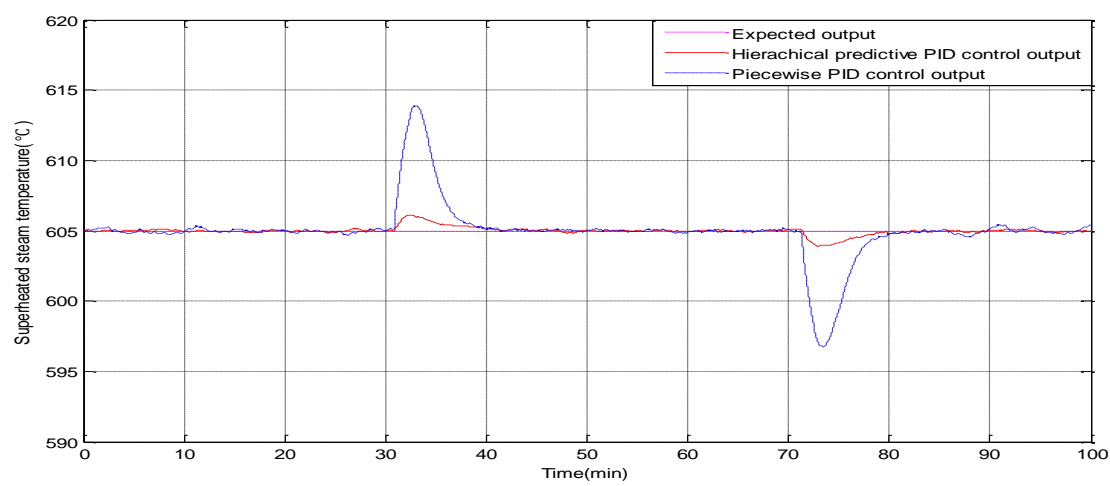

Figure 10. Output Curves of System Step Response with Internal Disturbance under Load that Decreases First and Increases Then

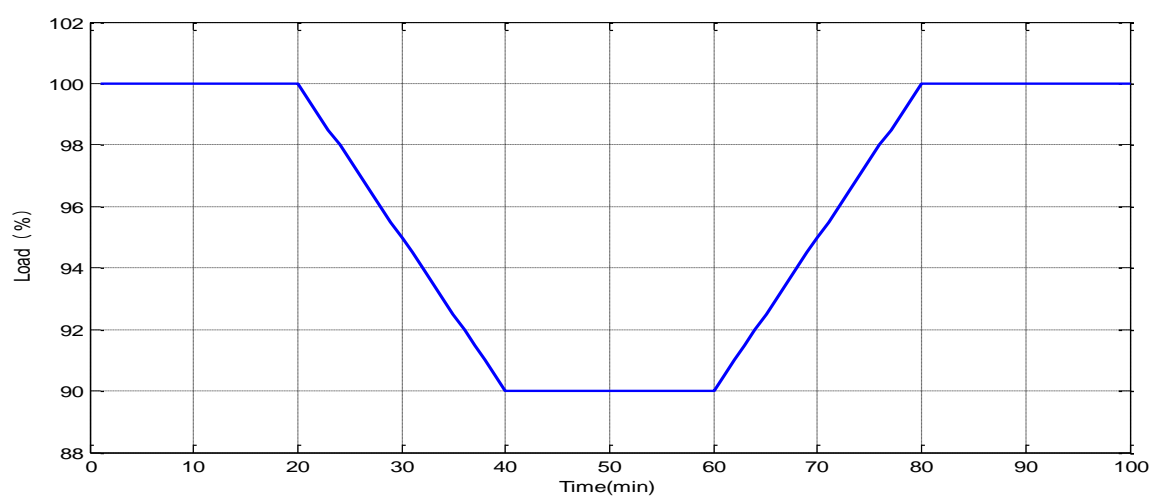

Figure 11. Variation Trend of Load that Decreases First and Increases Then 


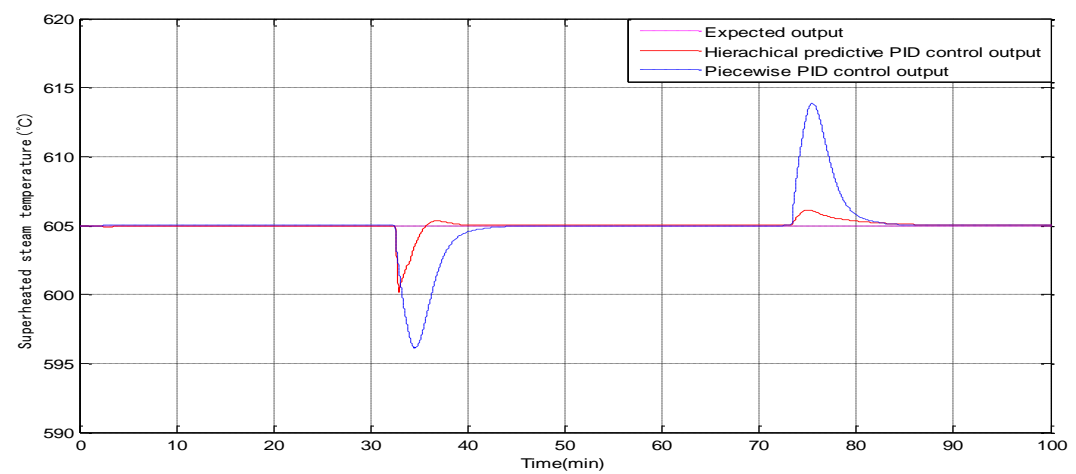

\section{Figure 12. Output Curves of System Step Response without Disturbance under Load that Decreases First and Increases Then}

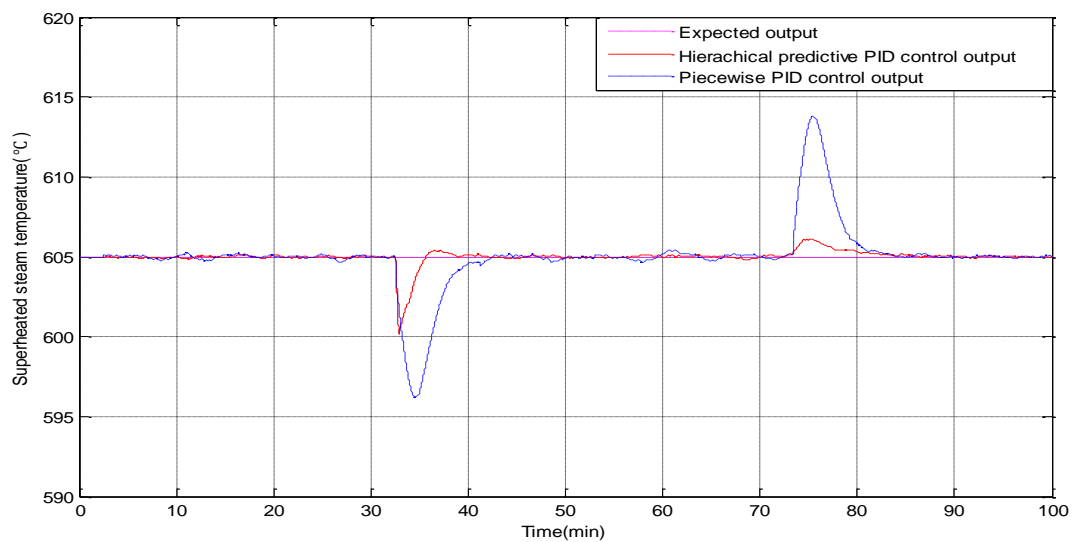

Figure 13. Output Curves of System Step Response with Internal Disturbance under Load That Decreases First and Increases Then

It can be seen from Figure 9 and Figure 12 that the hierarchical predictive PID control strategy makes smaller overshoot in the load transition process, which means the proposed method enables SST control system to have a better control effect when the load changes non-monotonically. Then the white noise is introduced in the simulation whose expectation is 0 and variance is 400 . It can be seen from Figure 10 and Figure 13 that as the minor loop of the proposed structure is qualified with a better anti-disturbance ability, the introduction of internal disturbance doesn't cause a large fluctuation of SST. From Figure 9, Figure 12, Figure 10 and Figure 13, it can be seen that the proposed method makes the settling time shorter in the load decreasing process. In conclusion, the hierarchical predictive PID control system has a better control performance and stronger anti-disturbance ability, which can adapt to the model change process of the SST under non-monotone load changes.

\section{Conclusion}

In order to solve the SST control problem of USC units under large-scale load changing, such as model uncertainty and lack of compatibility with advanced control algorithms, a hierarchical GPC-based PID control strategy is proposed in this paper. The cascade PID control strategy is applied in the bottom level to adapt to the characteristics of the DCS. The upper level consists of three modules - model parameters identification module, on-line optimization module and PID parameters 
tuning module. The performance index based on GPC is derived, and FFRLS based on rolling window is employed to identify the SST model. PID parameters tuning model is established based on predictive index. The simulation results show that the hierarchical predictive PID control method is qualified with shorter settling time and stronger robustness than piecewise PID control. It can adapt to the model change process of the SST. In this paper, the theoretical study and simulation analysis has obtained some achievements, but further research will need to be done to compare the proposed control method with other widely used SST control strategies, and to apply the method in DCS of USC units.

\section{References}

[1] Q. Zhu, J. Zhang and J. Wang, "Design Of Fuzzy and CMAC Parallel Controller Based On GA For Main Steam Pressure in Supercharged Boiler", Control and Decision Conference, CCDC, (2008), pp. 4934-4938.

[2] D. Ronan and O. Mark, "A New Approach To Quantify Reserve Demand in Systems With Significant Installed Wind Capacity", IEEE Transactions on Power Systems, vol. 20, no. 2, (2002).

[3] A. Chaibakhsh, A. Ghaffari and S. Moosavian, "A Simulated Model for A Once-Through Boiler by Parameter Adjustment Based on Genetic Algorithms", Simulation Modeling Practice and Theory, vol. 15, (2007), pp. 1029-1051.

[4] G.C. Goodwin, J.C. Aguiero and J.S. Welsh, "Robust Identification of Process Models From Plant Data", Journal of Process Control, vol. 18, no. 9, (2008), pp. 810-820.

[5] Y. Gao and E. Meng, "Online Adaptive Fuzzy Neural Identification and Control of a Class of MIMO Nonlinear Systems", IEEE Transactions on Fuzzy Systems, vol. 11, no. 4, (2003), pp. 462-477.

[6] S. Min, "On-line Generalized Predictive Control Combined with Recursive Least Squares System Identification", Ph.D. dissertation, Univ. Duke, United States, (2003).

[7] X. Shengyuan, L. James and C. Tongwen, "Robust Control For Uncertain Discrete Stochastic TimeDelay Systems”, Systems \& Control Letters, vol. 51, no. 3, (2004), pp. 203-215.

[8] J. Cike and A.W. Ordys, "Predictive Control For Industrial Applications", Annual Reviews in Control, vol. 25, (2001).

[9] D. Feng, C. W. Tong and Q. Li, "Bias Compensation Based Recursive Least- Squares Identification Algorithm for MISO Systems", IEEE Transactions on Circuits and Systems, pp. 349-353, (2006).

[10] Y.X. Su, Dong Sun and B.Y. Duan, "Design Of An Enhanced Nonlinear PID Controller", Mechatronics, vol. 15, no. 8, pp. 1005-1024, (2005).

[11] T. Yalcinoz and H. Altun, "Environmentally Constrained Economic Dispatch Via A Genetic Algorithm With Arithmetic Crossover", 6th Conf. IEEE AFRICON, pp. 923-928, (2003).

[12] J. Zeng, Y. Xie and L. Chen, "Design of Main Steam Temperature Cascade Control System Based on Fuzzy Self-Tuning PID Controller", Intelligent Computation Technology and Automation, 2008 International Conference on, (2008), 1.1:878-881.

[13] R. Yaoruan, Y. Changli, C. Huixin and L. Bin, "On-Line Order Estimation And Parameter Identification For Linear Stochastic Feedback Control Systems (CARMA Model)”, Automatica, vol. 39, no.2, pp. 243-253, (2003).

[14] M. Lentjes, K. Dickmann and J. Meijer, "Calculation And Optimization Of Sample Identification By Laser Induced Breakdown Spectroscopy Via Correlation Analysis", Spectrochimica Acta Part B: Atomic Spectroscopy, vol. 62, pp. 56-62, (2007).

[15] S.L. Kukreja, R.E. Kearney and H.L. Galiana, "A Least-Squares Parameter Estimation Algorithm For Switched Hammerstein Systems With Applications To The Vor", IEEE Transactions on Biomedical Engineering, vol. 52, pp. 431-444, (2005).

[16] X. Blasco, J. M. Herrero, M. Martínez and J. Senent, "Nonlinear Parametric Model Identification with Genetic Algorithms", Application to a Thermal Process. Lecture Notes in Computer Science, pp. 466473, (2001).

[17] M.A. Cavuslu, C. Karakuzu and F. Karakaya, "Neural Identification of Dynamic Systems on FPGA with Improved PSO Learning", Applied Soft Computing, vol.12, no.9, pp.2707-2718, (2012).

[18] H. Li and Z. Zhenyu, "The Application Of Immune Genetic Algorithm In Main Steam Temperature Of PID Control Of BP Network", Physics Procedia, vol.24, pp.80-86, (2012). 


\section{Authors}

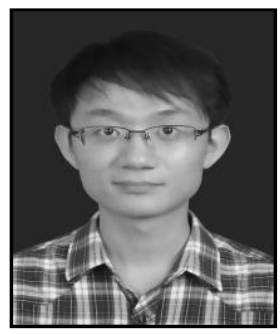

Xin Li, He received the B.S. degree in automation in 2011 from Wuhan University, Wuhan, China. He is currently pursuing the PhD degree from Department of Automation, Wuhan University, China. His research interests include advanced control, evolutionary algorithms, and their application in energy optimization.

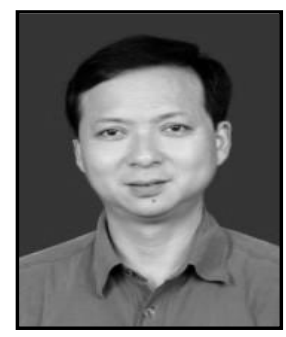

Yanjun Fang, He received the B.S. degree in thermal measurement and automation in 1982 from Wuhan University, Wuhan, China, and M.S. and Ph. D degrees in automation of electronic power system from Wuhan University, Wuhan, China, in1985and 1988, respectively. In 1992, he became a Research Fellow in Delft Hydraulic, Holland. Currently, he is a Professor in Department of Automation, Wuhan University, China, and became a $\mathrm{PhD}$ supervisor from 2000. His research interests include industrial automation, control theory and intelligent algorithms. He has won several awards including Cross-century Talents by the Ministry of Electric Power of China, 1997. He is the founder and director of Wuhan Automation Engineering Technology Research Center.

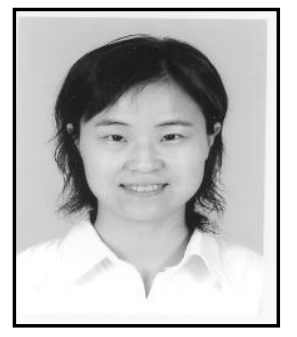

Rong Zhang, She received the B.S. and M.S. degrees in computer application from Wuhan University, Wuhan, China, in 1993 and 1998 respectively, and $\mathrm{PhD}$ degree in fluid machinery from Wuhan University, Wuhan, China, in 2010. As a visiting scholar, she got a research assistant position and worked in Department of Computer Science, National University of Singapore from 2004 to 2006. Her research interests include industrial automation, control theory and intelligent algorithms. 